\title{
Saliency Map Generation
}

\author{
Kiran Kumar M Mogalai ${ }^{1}$, Dr. Veenadevi S $\mathbf{V}^{2}$ \\ Dept. of Electronics and Communication, R.V College of Engineering, Bengaluru, India ${ }^{1}$ \\ Associate Prof, Dept. of Electronics \& Communication, R.V College of Engineering, Bengaluru, India ${ }^{2}$
}

\begin{abstract}
Saliency Map is the process of distinguishing the pixels from its neighboring pixels. Saliency Map plays a vital role in many computer vision and image processing applications. This paper briefs the effective use of Saliency Map in Automobile applications. There are many individual algorithm like pedestrian detection, sign board detection, lane detection, traffic board detection algorithm and so on, but the computational time will be more. Saliency Map acts as an intermediate stage and reduce the computational time. Bottom-up approach is used to extract the saliency of an image. Graph based SLIC segmentation is used to segment the foreground object from the background.
\end{abstract}

Keywords: Saliency Map, Graph based SLIC segmentation, foreground, background.

\section{INTRODUCTION}

Humans can quickly recognize the most noticeable object in the given image or scene, this is termed as Salient object. Focus all their attention on salient object neglecting the redundant region. Unlike, finding the salient object computationally is an challenging task in computer vision. Apart of Automobile application saliency map is also used in image segmentation, Object detection, Adaptive compression, Auto focusing, Object tracking and so on. Basically there are two approaches to extract the saliency map of an image namely Bottom-up approach and Top-down approach. In the bottom-up approach saliency is extracted by utilizing the parameters such as color, texture, contrast. These parameters are compared with the neighboring pixels and create the saliency map. In other words bottom-up approach there is no knowledge of the content present in an image. In Top-down approach saliency is based on template matching process that is we know what we are searching. The first step towards object recognition is object detection. Object detection aims at extracting an object from its background before recognition. But before performing recognitive feature analysis, how can a machine vision system extract the salient regions from an unknown background?

In today's growing technology accuracy and speed are the two most vital parameter. When it comes to real time application execution time is important. The driverless car, that is Autonomous car has to make the thousands of decision within fraction of second. In image processing, to identify an object the entire image has to be scanned and processed which is time consuming. Saliency map can be used in such cases were execution time is most important. Saliency map is an intermediate stage which helps to reduce the region of interest neglecting the redundant region. Fig.1.1 shows the example of sign board detection were sign board the region of interest and the remaining region is redundant which can be neglected.

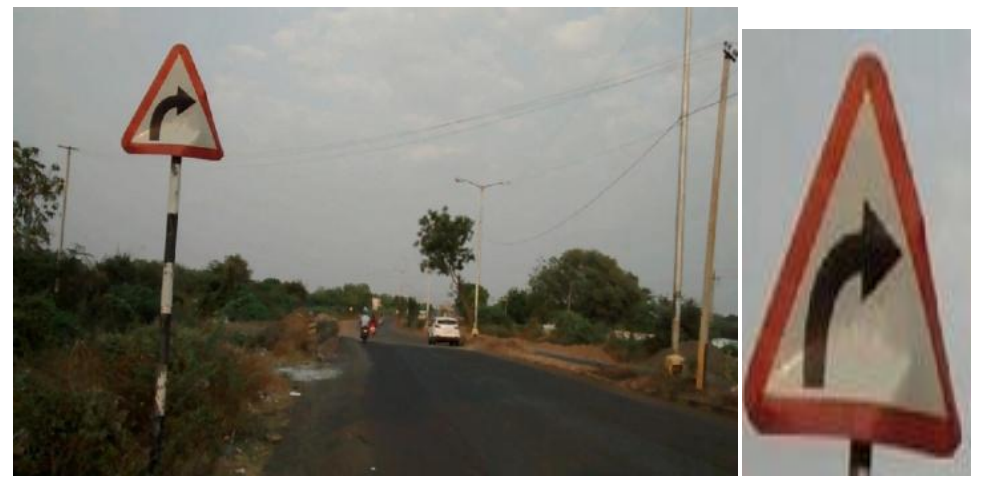

Fig.1.1 Input image for sign board detection (left) which consist of redundant region. Image which consist of only sign board which the region of interest (Right)

In recent years number of saliency models have been proposed, one of the earliest model was [1],[2] which involves no learning process, extracted the saliency from the filter output for each channel. Complex algorithm is used like Entropy [3], local contrast [4] and image difference [5]. Most of the above mentioned method utilizes the center contrast and different in feature in an entire image. 


\section{ALGORITHM}

\section{A. Flow chart of Algorithm}

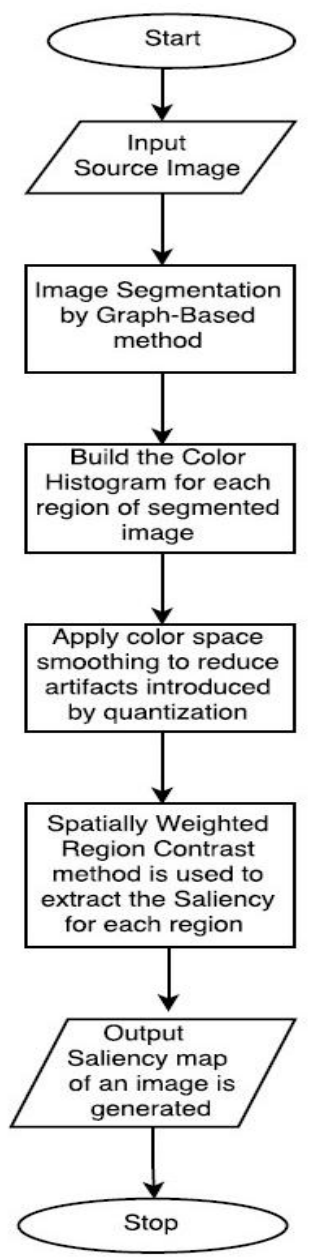

Fig.2.1 Algorithm flow.

\section{B. Image Segmentation}

In computer vision, image segmentation is the process of partitioning a digital image into multiple segments. More precisely, image segmentation is the process of assigning a label to every pixel in an image such that pixels with the same label share certain characteristics. In graph based algorithms, each pixel is treated as a node in a graph, and edge weight between two nodes are set proportional to the similarity between the pixels. Superpixel segments are extracted by effectively minimizing a cost function defined on the graph. Fig.2.2 shows the segmented image . The Graph based algorithm performs an agglomerative clustering of pixel nodes on a graph, such that each segment, or superpixel, is the shortest spanning tree of the constituent pixels.

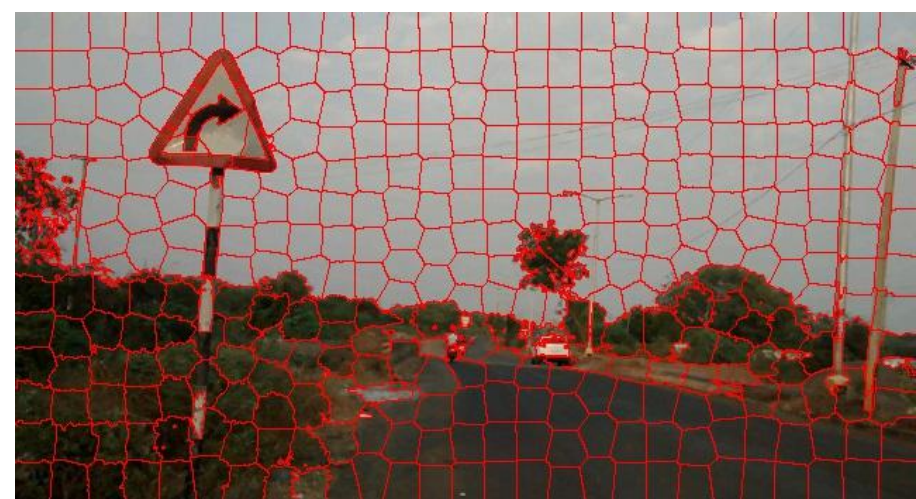

Fig.2.2 The output of image segmentation algorithm 


\section{Histogram}

Naively evaluating the saliency value for each image pixel using takes more time, which is computationally too expensive even for medium sized images. Thus, the key to speed up is to reduce the number of pixel colors in the image. However, the true color space contains 256 power of 3 possible colors, which is typically larger than the number of image pixels. Further reduce the number of colors by ignoring less frequently occurring colors. By choosing more frequently occurring colors and ensuring these colors cover the colors of more than $95 \%$ of the image pixels. Hence histogram is mainly used to reduce the number of image, decreasing the computational time.

\section{Color Space Smoothing}

Smoothing is the processes of removing or reducing the noisy component from an image, generated during intermediate stages. Although we can efficiently compute color contrast by building a compact color histogram using color quantization and choosing more frequent colors, the quantization itself may introduce artefacts. Some similar colors may be quantized to different values. In order to reduce noisy saliency results caused by such randomness, we use a smoothing procedure to refine the saliency value for each color.

\section{E. Assigning the Saliency}

The RGB color space is converted into $\mathrm{L} * \mathrm{a} * \mathrm{~b}$ color space because it provide the human vision interpretation. First we should calculate the distance of each pixel to the rest of pixels in the same frame. And then add all of this minus value together. We use I as the representative of the pixel value. Then we get the following equation:

$$
\operatorname{SAL}\left(\mathrm{I}_{\mathrm{k}}\right)=\sum\left|\mathrm{I}_{\mathrm{k}}-\mathrm{I}_{\mathrm{i}}\right|
$$

Ii is the rest of pixel value in current frame, except $I_{k}$. Besides, the value of $I_{k}$ is in the range of $[0,255]$. The following equation is the expanded form of this equation.

$$
\operatorname{SAL}\left(I_{k}\right)=\left|I_{k}-I_{1}\right|+\left|I_{k}-I_{2}\right|+\ldots+\left|I_{k}-I_{N}\right| \ldots \ldots
$$

Where $\mathrm{N}$ is the total number of pixels in the current frame. Then we can further restructure our formula. We put the value that has same I together.

$$
\operatorname{SAL}\left(\mathrm{I}_{\mathrm{k}}\right)=\sum \mathrm{F}_{\mathrm{n}} *\left|\mathrm{I}_{\mathrm{m}}-\mathrm{I}_{\mathrm{n}}\right| \ldots
$$

Which $F_{n}$ is the frequency of $I_{n}$. And the value of $n$ belongs to $[0,255]$. The frequencies is expressed in the form of histogram, and the computational time of histogram is $\mathrm{O}(\mathrm{N})$ time complexity.

The Fig.2.3 shows the saliency image. Further the output we can observe that only the sign board is highlighted which is the region of interest in sign board detection.

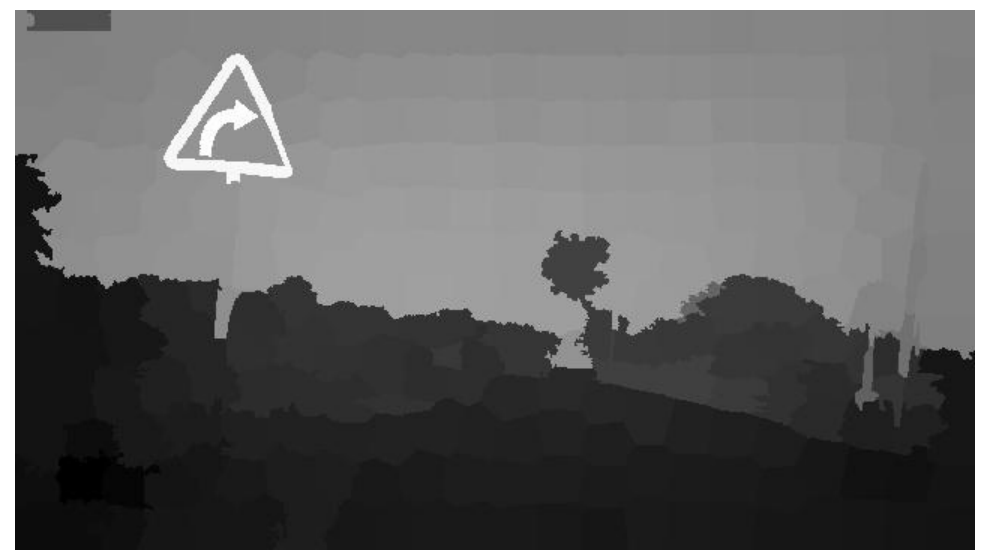

Fig.2.3 Saliency of an input image

\section{RESULTS}

The Fig3.1 shows the results of the intermediate stages and the final saliency of an input image. The proposed algorithm is evaluated on few data sets with the system specification desktop PC with Intel Pentium Dual Core - 2140 $1.6 \mathrm{GHz}$ CPU and 2GB RAM. 

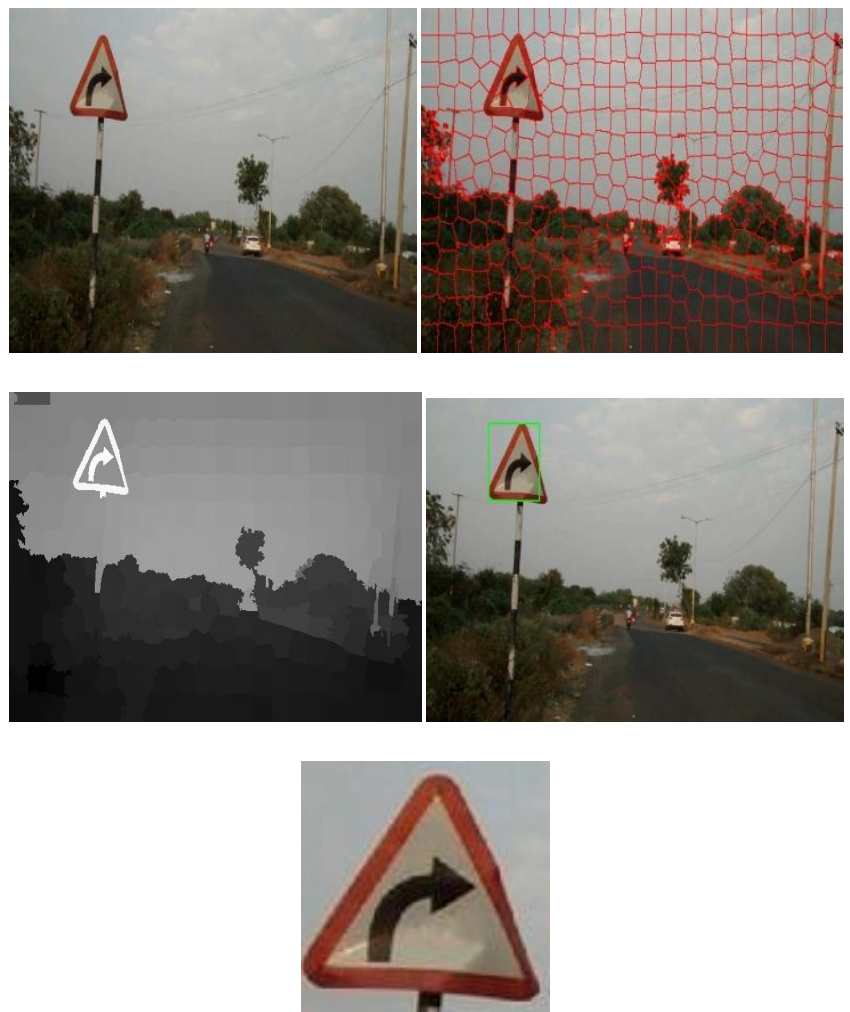

Fig.3.1 First row images, Input image (left) and segmented image (right). Second row, saliency image (left) and Region of Interest (right). Last row represents the salient object being isolated.

\section{CONCLUSION AND FUTURE WORK}

In this paper we have proposed an algorithm to detect salient regions in images. The proposed algorithm generates spatially consistent high quality saliency maps at the cost of reduced computational efficiency. There exists a variety of applications saliency in computer vision and this paper explains about the use of saliency in automobile to increase the processing speed. In the future we intend to learn the benefits of this saliency in more applications, such as adaptive image compression, auto focussing, object detection, image retrieval.

\section{REFERENCES}

[1] R. Achanta, S. Hemami, F. Estrada, and S. Susstrunk. Frequencytuned salient region detection. In CVPR, 2009

[2] K.-Y. Chang, T.-L. Liu, H.-T. Chen, and S.-H. Lai. Fusing generic objectness and visual saliency for salient object detection. In ICCV, 2011.

[3] J. Feng, Y.Wei, L. Tao, C. Zhang, and J. Sun. Salient object detection by composition. In ICCV, 2011

[4] M. M. Cheng, G. X. Zhang, N. J. Mitra, X. Huang, and S. M. Hu. Global contrast based salient region detection. In CVPR, 2011.

[5] T. H. Kim, K. M. Lee, and S. U. Lee. Learning full pairwise affinities for spectral segmentation. In CVPR, 2010.

[6] Y. Lu, W. Zhang, H. Lu, and X. Y. Xue. Salient object detection using concavity context. In ICCV, 2011.

[7] J. Han, K. Ngan, M. Li, and H. Zhang. Unsupervised extraction of visual attention objects in color images. IEEE TCSV, 16(1):141-145, 2006.

[8] Y. Zhai and M. Shah. Visual attention detection in video sequences using spatiotemporal cues. In ACM Multimedia, pages 815-824, 2006

[9] S. Goferman, L. Zelnik-Manor, and A. Tal. Context-aware saliency detection. In CVPR, pages 2376-2383, 2010.

[10] Y.-F. Ma and H.-J. Zhang. Contrast-based image attention analysis by using fuzzy growing. In ACM Multimedia, pages 374-381, 2003

[11] Delaitre, J. Sivic, and I. Laptev. Learning person-object interactions for action recognition in still images. In NIPS, 2011. 